\title{
Generación de señales de electroestimulación percutánea para terapias pasivas en rehabilitación neuromuscular de tobillo empleando lógica difusa
}

\author{
Miriam C. Reyes-Fernández, Ruben Posada-Gomez, Albino Martinez-Sibaja,
} Alberto A. Aguilar-Lasserre, Juan C. Trujillo-Caballero

División de Estudios de Posgrado e Investigación del Instituto Tecnológico de Orizaba Orizaba, Veracruz, México

mcreferlgmail.com, rposadaditorizaba.edu.mx, albino3_mx@yahoo.com, albertoaal@hotmail.com, juan_carlostrujillo@hotmail.com

\begin{abstract}
Resumen. Este artículo describe el desarrollo de un sistema de electroestimulación percutánea para terapias pasivas, que pueda mejorar los procesos de rehabilitación aplicados a los pacientes con esguince en el tobillo, mediante el empleo de señales de electroestimulación para mejorar el impacto de los ejercicios y favorecer la retroalimentación entre el profesional y el paciente, logrando de esta manera una recuperación más rápida y eficaz. Se plantea el empleo de la Lógica Difusa como herramienta para determinar el tipo de señales más convenientes a usar en el tratamiento del paciente tomando la experiencia del especialista dependiendo de las características del paciente así como del tipo de lesión. Así mismo se sientan las bases para el desarrollo de los protocolos de experimentación correspondientes para algunos casos de estudio.
\end{abstract}

Palabras clave: Rehabilitación pasiva, electroestimulación percutánea, rehabilitación de tobillo.

\section{Introducción}

Las lesiones de tobillo son comunes a lo largo de la vida de un ser humano, sin importar condiciones de vida, si hacen o no deporte, edad, peso, etc. Ocurren en cualquier momento, y suelen ser dolorosas y de recuperación a mediano o largo plazo. Según la Secretaría del Trabajo y Previsión Social [1], las lesiones del tobillo y pie son el segundo tipo de lesiones más frecuentes después de las lesiones de muñeca y mano (ver Fig. 1).

Con el fin de evitar que las lesiones que una persona sufra lo lleven a una discapacidad motora permanente, es necesario que esta lleve un proceso de rehabilitación. El cual, dependiendo del tipo de lesión consiste en la una serie de ejercicios y tratamientos diseñados por el médico traumatólogo en conjunto con un equipo de terapeutas en rehabilitación motriz. Los tratamientos sugeridos pueden clasificarse de manera global en dos tipos [2]: 
- Rehabilitación activa: Consiste en una serie de ejercicios que el paciente debe hacer permitiendo que se realice el movimiento de las articulaciones afectadas, el terapeuta orienta al paciente para la realización de la rutina y generalmente se realiza precozmente tras los primeros días del episodio, generalmente en centros especializados.

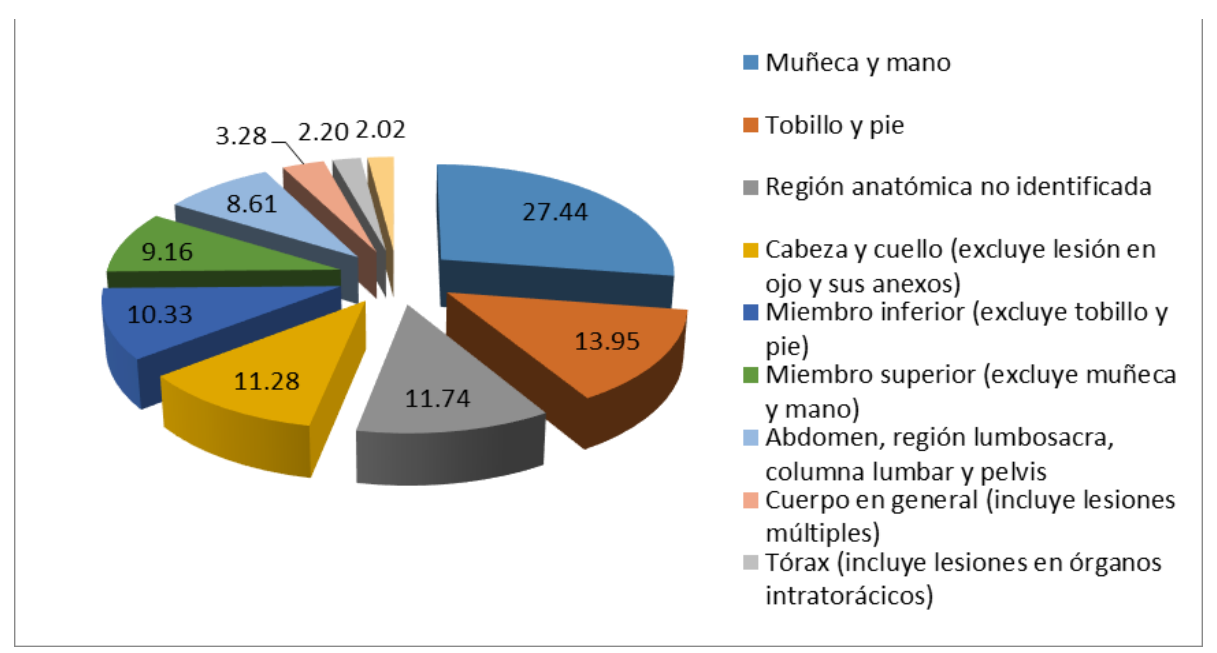

Fig. 1. Accidentes de Trabajo Según Región Anatómica y Sexo, 2010 - 2012 Nacional (Datos obtenidos de [1]).

- $\quad$ Rehabilitación pasiva: Consiste en la movilización por parte del terapeuta de las articulaciones afectadas tres o cuatro veces al día, para prevenir contracturas musculares y lesiones articulares.

En ambos casos se cuentan con diferentes herramientas que ayudan a la realización de este tipo de terapias. En el caso de la rehabilitación activa, la mecanoterapia es la herramienta más frecuentemente utilizada [3]; Por otro lado, en el caso de la rehabilitación pasiva se reporta que los movimientos asistidos produzcan una cierta relajación de las estructuras fibrosas y de esta manera se mantienen activos las estructuras afectadas reduciendo las contracturas y retracciones musculares, en estas circunstancias recientemente se han efectuado trabajos que sugieren que la electroestimulación es una herramienta recomendable para recuperar la movilidad [4].

De la misma manera tanto la terapia de rehabilitación activa como la pasiva presentan diferentes ventajas para la recuperación de la movilidad, sin embargo estas técnicas de rehabilitación se aplican de manera alternada. En los casos en los que el paciente tiene muy poca movilidad o no es capaz de efectuar los movimientos por sí mismo se aplica la rehabilitación pasiva y el uso de la electroestimulación juega el papel de prevención de lesiones articulares por inmovilidad; cuando el paciente tiene movilidad suficiente para efectuar los movimientos por sí mismo, continua su tratamiento en una sala de mecanoterapia, donde los aparatos mecánicos tienen la función de limitar el movimiento que se efectúa durante las terapias. 


\section{Lesiones del tobillo}

El tobillo es la parte de la anatomía humana donde se unen el pie y la pierna. La pieza superior está conformada por la tibia y el peroné, huesos de la pierna, y por el astrágalo, que forma parte del pie.

El tobillo es el responsable de la marcha y de los movimientos que se hacen al andar así como de permitir que el ser humano pueda mantener una postura erguida.

Al tener una función tan importante para el movimiento es común que esta articulación sufra lesiones que van de leves a severas, ya que continuamente está expuesta a diversos accidentes que derivan en fracturas o esguinces [5]. Estas lesiones conllevan dolor, inmovilización y un tiempo de recuperación que varía de acuerdo al tipo, a las condiciones del paciente y a las condiciones de rehabilitación con que se cuenten, en este sentido existen diversos estudios enfocados a este tipo de lesiones principalmente en el ámbito de la medicina del deporte [6].

Una persona puede sufrir un esguince de tobillo a cualquier edad y casi cualquier circunstancia de marcha, sin embargo, hay grupos con más riesgo, como los deportistas o las personas de la tercera edad; los primeros, porque someten sus cuerpos a pruebas físicas extremas, los segundos, porque el sistema óseo se encuentra deteriorado y muchas veces también está resentida la articulación por el paso del tiempo y por accidentes previos.

\subsection{Tipos de lesiones}

El tobillo puede resistir impactos, pero cuando exceden el límite de resistencia de los ligamentos o de los huesos, se presentan fracturas o esguinces que deben ser atendidas inmediata y adecuadamente, de lo contrario, podrían presentarse secuelas que podrían incluso llevar a una discapacidad permanente.

En una lesión del tobillo intervienen tanto la dirección como la magnitud de las fuerzas y la posición del pie y el tobillo durante el traumatismo generalmente en flexión plantar por lo que la inflamación y el dolor suelen estar presentes en el Fascículo Peroneo-astragalino Anterior (FPAA), en el Fascículo Peroneo-calcáneo (FPC) y el Fascículo Peroneo-astragalino Posterior (FPAP) [7].

Tabla 1. Sistema de clasificación para la descripción de los grados de esguince del ligamento lateral externo del tobillo.

\begin{tabular}{|c|c|c|c|}
\hline Características clínicas & Grado 1 & Grado 2 & Grado 3 \\
\hline Localización del dolor & FPAA & FPAA,FPC & FPAP,FPC,FPAA \\
\hline Edema, Equimosis & $\begin{array}{c}\text { Local y escasamente } \\
\text { notable }\end{array}$ & $\begin{array}{l}\text { Local y } \\
\text { moderadamente } \\
\text { notable }\end{array}$ & $\begin{array}{c}\text { Difuso (varios } \\
\text { segmentos del } \\
\text { complejo articular) } \\
\text { muy notable }\end{array}$ \\
\hline Capacidad de carga & Completa o parcial & $\begin{array}{l}\text { Dificultosa sin } \\
\text { muletas }\end{array}$ & $\begin{array}{l}\text { Incapacidad } \\
\text { funcional total }\end{array}$ \\
\hline Daño ligamentoso & Elongación & Ruptura parcial & Ruptura total \\
\hline Inestabilidad & No presenta & $\begin{array}{c}\text { Moderada (escasa) o } \\
\text { no presenta }\end{array}$ & Inestabilidad total \\
\hline
\end{tabular}


El esguince de tobillo es una de las lesiones más frecuentes, consiste en un estiramiento que hace que el ligamento supere su límite de elasticidad. Un esguince se produce por apoyar mal el pie, caídas o flexiones muy fuertes, movimientos bruscos al hacer deportes, y una enorme variedad de movimientos que pueden provocarla. Esto produce que se estiren los ligamentos de manera extraordinaria provocando inflamación, hematomas y la dificultad para andar o ponerse de pie. La Tabla 1, muestra la clasificación según el grado de esguince en el tobillo [8].

La rehabilitación de un esguince suele ser más larga de lo que la mayoría de los pacientes espera. El período de recuperación tras el tiempo de reposo suele ser en promedio de 15 días para los esguinces de grado I; 44 días para el grado II; y de 62 días para los esguinces de grado III [7].

\subsection{Rehabilitación del tobillo}

Una vez que el tobillo se ha lesionado, lo primero es inmovilizar la articulación para evitar afectaciones mayores, acto seguido es necesaria la evaluación médica para descartar (o confirmar) fracturas.

De confirmarse el diagnóstico de esguince en el tobillo, se procede a evaluar el grado del mismo, de acuerdo a la siguiente clasificación:

- Grado I o leve: hay estiramiento de las fibras y lesiones microscópicas. No existe inestabilidad de la articulación y, en general permite caminar con molestias.

- Grado II, con rotura parcial de algunas fibras. Generalmente el dolor y la hinchazón aparecen rápido, y se acompaña de dolor moderado o severo; existe cierta inestabilidad y es imposible mantener el equilibrio. Es frecuente que aparezca una equimosis, un hematoma en la parte lateral del pie a los pocos días, como consecuencia del sangrado al romperse las fibras.

- Grado III, con rotura completa del ligamento, y en ocasiones con arrancamiento óseo. Existe dolor severo, inflamación importante, inestabilidad e incapacidad para el apoyo. Requiere atención por el traumatólogo y en algunos casos incluso cirugía.

Una vez determinado el tipo de esguince, se procede a inmovilizar mediante férula al tobillo, y la prescripción suele ser reposo, medicamentos contra la inflamación y el dolor y regreso a consulta médica.

Después de un tiempo que varía de acuerdo al grado de la lesión, el paciente puede comenzar a hacer movimientos de estiramiento en casa, para obtener de nuevo fuerza y tonicidad muscular. De ser necesario, también estará recibiendo la ayuda del experto para usar máquinas de movimiento pasivo.

Un tobillo con esguince puede requerir entre 2 y 6 semanas para recobrarse, pero los esguinces más graves pueden tardar en sanar hasta 12 semanas.

\section{Desarrollo del electroestimulador}

La electroestimulación es una técnica que se emplea de manera paralela al tratamiento de rehabilitación motriz principalmente para la reducción de la espasticidad. Lisinski et al [9] presentan un estudio de pacientes que sufrieron un 
accidente cerebrovascular y que han sido tratados mediante la electroestimulación y muestran la efectividad del tratamiento para la tonicidad como para la fuerza muscular las cuales son introducidas en la muñeca y tobillo del paciente. Para evaluar la actividad muscular se emplearon las mediciones de electromiograma encontrándose un aumento significativo en la mayoría de los pacientes.

Amiridis et al [10] presentan un estudio realizado en adultos mayores empleando electroestimulación para determinar el efecto de la flexión dorsal del tobillo. Se monitoreo el comportamiento de los pacientes después de efectuar las sesiones de electroestimulación para efectuar tareas de balance como estar parados con una sola pierna, y posturas normales cuantificando las variaciones que se obtenían en el rango de oscilación de las personas. Los autores concluyen en base a estos datos que las sesiones de electroestimulación son benéficas para pacientes no muestran una discapacidad unilateral como en los pacientes que han sufrido algún accidente cerebrovascular.

La utilización de electroestimulación con fines terapéuticos en el área de la rehabilitación continúa siendo un tema de interés para el desarrollo de prototipos como lo muestra la patente de Skahan et al [11] en la cual se presenta un sistema de electroestimulación alojado en un guante. El objetivo es que el guante desarrollado sirva al mismo tiempo como un sistema de electroestimulación y pueda transmitir las señales de acoplamiento con el usuario.

\subsection{Parámetros de la electroestimulación}

Para llevar a cabo la estimulación eléctrica neuromuscular existen diferentes parámetros de estimulación tales como la intensidad, forma de onda, anchura de pulso, frecuencia, etc. que son variables que deben ser tomadas en cuenta para la reproducción de los resultados esperados, ya que estos parámetros influirán considerablemente en los efectos musculares. Así por ejemplo Snyder-Mackler et al [12] reportan un aumento de fuerza al emplear señales de $75 \mathrm{~Hz}$, diferente a la reportada por Lieber et al. [13] y Paternostro-Sluga et al. [14] Quienes emplean pulsos de frecuencias de entre 30-50Hz.

Para este trabajo se han considerado efectuar variaciones en la frecuencia de los pulsos determinada directamente por el número de pulsos y la duración de los mismos. Posteriormente se pretende efectuar diferentes pruebas modificando la amplitud de los pulsos.

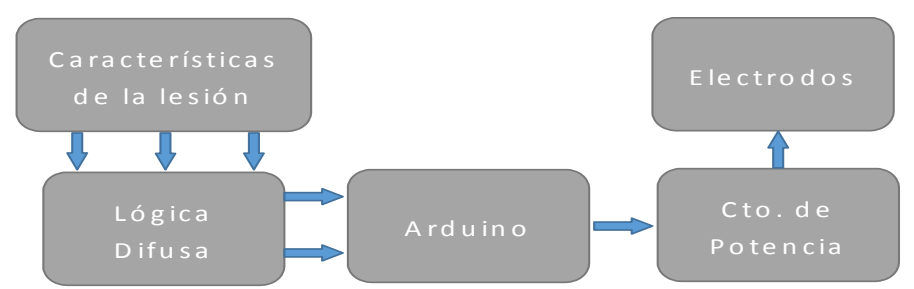

Fig. 2. Diagrama a bloques del sistema de electroestimulación desarrollado.

El electroestimulador desarrollado (Figura 2), genera los pulsos de voltajes elevados y bajas corrientes hacia los electrodos. Toma como entradas el número de pulsos y la 
duración de los mismos. Estos parámetros son proporcionados por el sistema difuso que genera estos datos a partir de la información del paciente respecto al dolor que refieran (Dolor), su capacidad de carga (Cap.Carga) y la estabilidad al caminar (Estabilidad).

De esta manera el sistema difuso que se desarrolló (Figura 3) toma como entradas las variables que representan el dolor, la capacidad de carga y la estabilidad durante la marcha, y como salidas el número de pulsos (N.Pulsos) y la duración de los pulsos (T.A.).

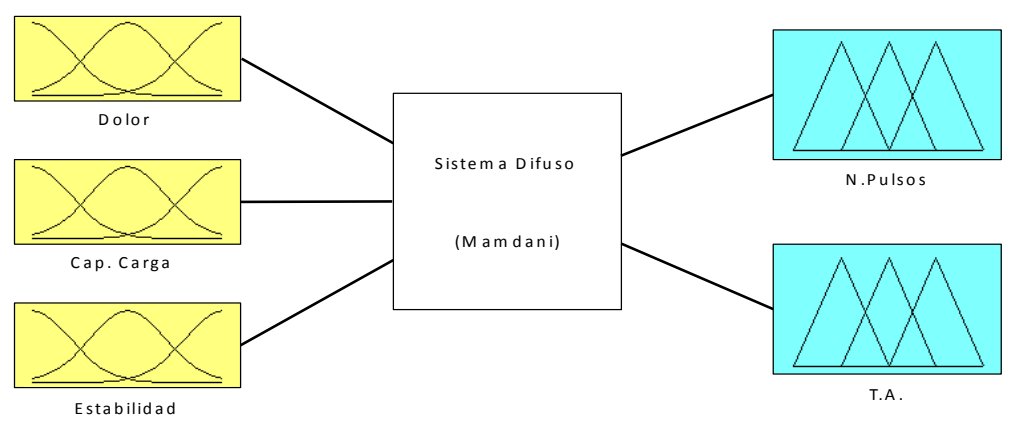

Fig. 3. Sistema difuso desarrollado.

\subsection{Definición de las variables lingüísticas}

El modelo de lógica difusa para la toma de decisiones tiene como propósito tomar una decisión basada en un conjunto de reglas (motor de inferencia) dadas por la experiencia del terapeuta de acuerdo a los valores de cada variable de entrada. En este caso las variables identificadas como entrada al modelo de lógica difusa son:

- Dolor. Al inicio de la sesión, el paciente le refiere al terapeuta el dolor que percibe en el miembro a tratar en un rango de 0 a 10, el cual puede ser identificado como "leve", "moderado" o "agudo". Esta entrada es definida como gaussiana con una apertura de 1.5 y centrada en 0,5 y 10 respectivamente.

- Cap.Carga. El terapeuta evalúa la capacidad del paciente para sostenerse de pie mientras sostiene su propio peso en un rango de 0 a 10 , esta variable se define de tipo gaussiana con una apertura de 1.5 y se identifica como "completa" $(0)$, si el paciente puede mantenerse de pie sin dificultad, "dificultosa" (5) si lo hace refiriendo molestias o "imposibilidad" (10) si no es posible que se sostenga de pie.

- Estabilidad. Para determinar la estabilidad, el terapeuta solicita al paciente que camine mientras el terapeuta observa la forma en que efectúa su marcha. El rango de esta variable de tipo gaussiana se encuentra entre 0 y $10 \mathrm{y}$ se identifica como "S/Problemas" (0) si el paciente puede desplazarse sin ayuda, "C/Dificultad" (5) si requiere de algún tipo de ayuda para desplazarse e "Imposibilidad" (10) si el paciente no puede desplazarse.

De la misma manera, los parámetros obtenidos por las variables de inferencia generan dos tipos de salidas difusas: 
- N.Pulsos. El tratamiento que el terapeuta efectuará en el paciente con el electroestimulador, envía un número de pulsos que pueden ir de 0 a 50 y son definidos como "pocos" (de 0 a 15), "medios" (15 a 35) o "muchos" (35 a 50). Esta salida es de tipo triangular con un máximo en 7.5, 25 y 42.5. El valor obtenido es redondeado al entero más cercano para ser aplicado por el sistema electroestimulador.

- T.A. Es el tiempo que el pulso estará en Alto, y este puede se encuentra en un rango de entre 0 y $250 \mu$ s. se define como "MuyCorta" (0 a 50), "Corta" (50 a 100), "Media" (100 a 150), "Larga" (150 a 200) y "MuyLarga" (200 a 250). De la misma manera el valor de salida debe ser redondeado al entero más próximo para poder ser empleado en el electroestimulador.

El mecanismo de inferencia toma los grados de pertenencia de cada entrada y genera una salida difusa en base al conjunto de reglas difusas (Tabla 2) resumidas en forma lingüística y que contienen el conocimiento del experto. Se toman en cuenta solo aquellas condiciones que el experto considera factibles y válidas. De esta manera por ejemplo no se evalúan aquellas condiciones en los que el paciente no tiene estabilidad y pueda tener una capacidad de carga completa, dado que es una condición que no se encuentra en la realidad.

Tabla 2. Reglas de inferencia empleadas en el sistema difuso.

\begin{tabular}{lll}
\hline$\#$ & \multicolumn{1}{c}{ Regla } & \multicolumn{1}{c}{ Salida } \\
\hline 1 & Dolor=Leve \& Cap.Carga=Comp. \& Estabilidad=S/Prob. & N.Pulsos => Pocos, T:A => MuyCorta \\
2 & Dolor=Leve \& Cap.Carga=Comp. \& Estabilidad=C/Dif. & N.Pulsos => Pocos, T:A => Corta \\
3 & Dolor=Leve \& Cap.Carga=Dif. \& Estabilidad=C/Dif. & N.Pulsos => Pocos, T:A => Media \\
4 & Dolor=Leve \& Cap.Carga=Dif. \& Estabilidad=Imp. & N.Pulsos => Pocos, T:A => Larga \\
5 & Dolor=Leve \& Cap.Carga=Imp. \& Estabilidad=C/Dif. & N.Pulsos => Pocos, T:A => Larga \\
6 & Dolor=Leve \& Cap.Carga=Imp. \& Estabilidad=Imp. & N.Pulsos => Pocos, T:A => MuyLarga \\
7 & Dolor=Leve \& Cap.Carga=Comp. \& Estabilidad=S/Prob. & N.Pulsos => Medios, T:A => MuyCorta \\
8 & Dolor=Leve \& Cap.Carga=Comp. \& Estabilidad=C/Dif. & N.Pulsos $=>$ Medios, T:A => Corta \\
9 & Dolor=Leve \& Cap.Carga=Dif. \& Estabilidad=C/Dif. & N.Pulsos => Medios, T:A => Media \\
10 & Dolor=Leve \& Cap.Carga=Dif. \& Estabilidad=Imp. & N.Pulsos => Medios, T:A => Larga \\
11 & Dolor=Leve \& Cap.Carga=Imp. \& Estabilidad=C/Dif. & N.Pulsos => Medios, T:A => Larga \\
12 & Dolor=Leve \& Cap.Carga=Imp. \& Estabilidad=Imp. & N.Pulsos => Medios, T:A => MuyLarga \\
13 & Dolor=Leve \& Cap.Carga=Comp. \& Estabilidad=S/Prob. & N.Pulsos => Muchos, T:A => MuyCorta \\
14 & Dolor=Leve \& Cap.Carga=Comp. \& Estabilidad=C/Dif. & N.Pulsos => Muchos, T:A => Corta \\
15 & Dolor=Leve \& Cap.Carga=Dif. \& Estabilidad=C/Dif. & N.Pulsos => Muchos, T:A => Media \\
16 & Dolor=Leve \& Cap.Carga=Dif. \& Estabilidad=Imp. & N.Pulsos => Muchos, T:A => Larga \\
17 & Dolor=Leve \& Cap.Carga=Imp. \& Estabilidad=C/Dif. & N.Pulsos => Muchos, T:A => Larga \\
18 & Dolor=Leve \& Cap.Carga=Imp. \& Estabilidad=Imp. & N.Pulsos => Muchos, T:A => MuyLarga \\
\hline
\end{tabular}

Los resultados de la evaluación de las reglas se interpretan para obtener una salida con un valor preciso. Para ello se emplea el modelo del centro de gravedad. Como puede verse en la figura 4. Si se toma un caso en el que el paciente presente Dolor moderado con un valor de 5; Tenga una capacidad de carga dificultosa con un valor de 
Miriam Reyes-Fernandez, Ruben Posada-Gomez, Albino Martinez-Sibaja, et al.

5 y presente dificultad para su estabilidad también con un valor de 5 . El sistema genera una salida correspondiente a 25 pulsos con una duración de $125 \mu$ s.

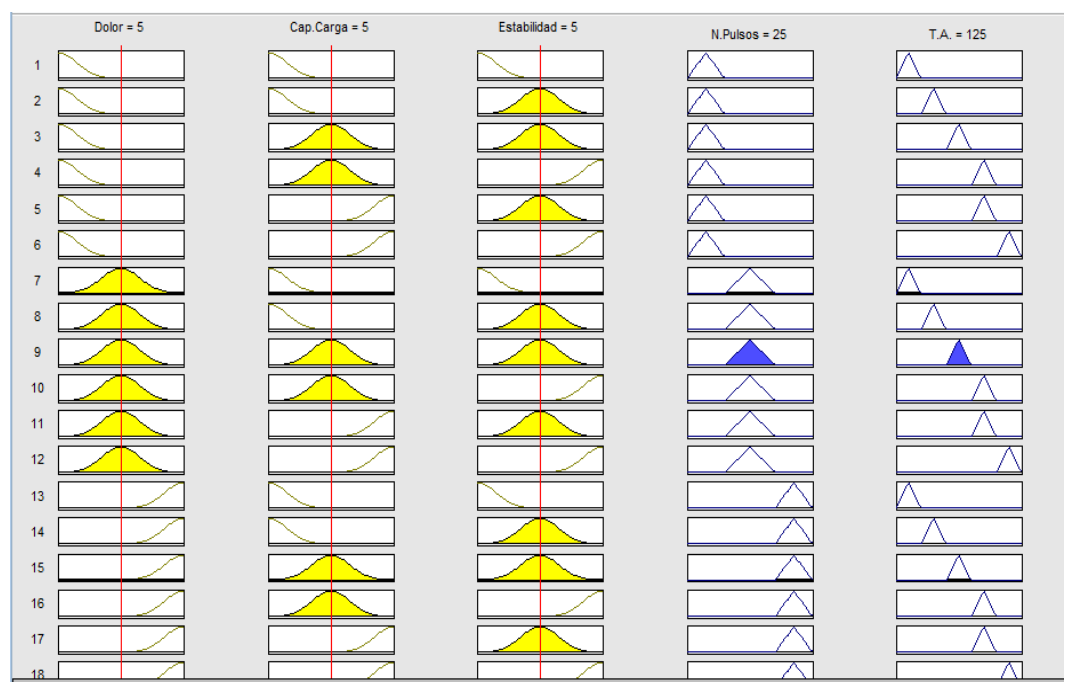

Fig. 4. Sistema difuso desarrollado.

\section{Resultados obtenidos}

Con el sistema desarrollado se generaron las señales de electroestimulación para diferentes casos. Los pulsos generados se muestran en la figura 5 y el código implementado en el Arduino puede verse en el Algoritmo 1.

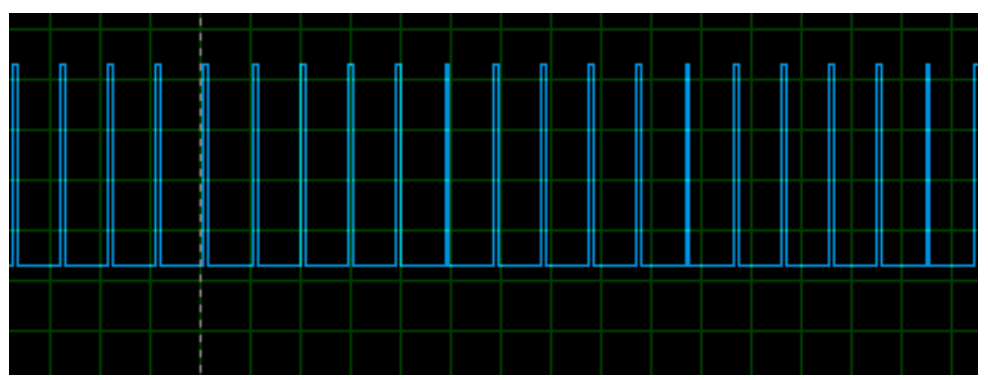

Fig. 5. Pulsos generados por el electroestimulador.

Algoritmo 1. Frangmento del programa para el Arduino empleado en el electroestimulador desarrollado.

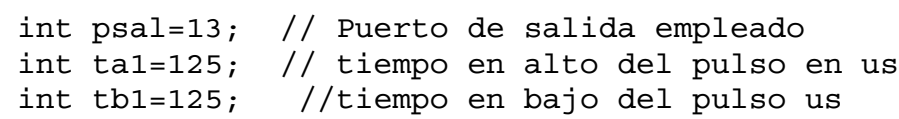




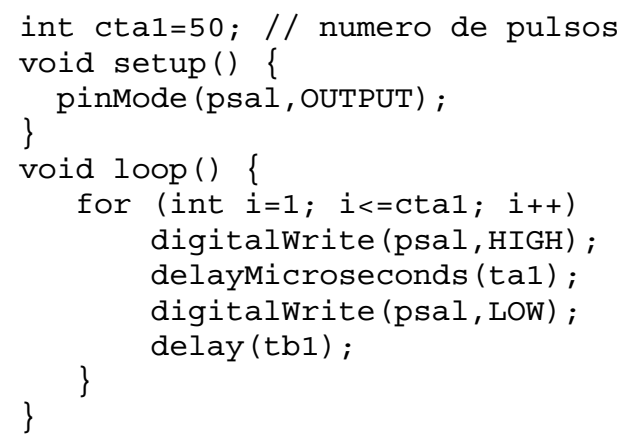

\section{Conclusiones y trabajos futuros}

La electroestimulación es una herramienta que puede resultar de gran utilidad en la rehabilitación motriz de los pacientes, es importante sin embargo que se efectúen mayores estudios respecto a los resultados que esta técnica producen en los pacientes, ya que se ha visto mejoría sobre todo en lo concerniente a la reducción del dolor referida pos los pacientes. Es necesario que se continúe con trabajos que permitan validar el empleo de la electroestimulación de una manera objetiva. El empleo de la lógica difusa resulta muy adecuado ya que permite decidir las características del tratamiento que se dará al paciente en cuanto a la duración y amplitud de las señales, tomando como base el conocimiento del experto y la sintomatología del paciente.

Como trabajos futuros, se implementará esta metodología también en la amplitud de la señal y se llevará a cabo un estudio que permita efectuar un seguimiento en los pacientes que empleen la electroestimulación con determinadas lesiones del tobillo.

\section{Referencias}

1. STPS, Información sobre Accidentes y Enfermedades de Trabajo Nacional 2003-2012. México (2012)

2. D. K. Spierer, R. Goldsmith, D. A. Baran, K. Hryniewicz, and S. D. Katz: Effects of active vs. passive recovery on work performed during serial supramaximal exercise tests. Int. J. Sport. Med., vol. 25, no. 2, pp. 109-14 (2004)

3. Araújo, M. E. A. D., Silva, E. B. D., Vieira, P. C., Cader, S. A., Mello, D. B. D., Dantas, E. H. M.: Reduction of the chronic pain associated to the scoliosis non structural in university students submitted to the Pilates method. Motriz: Revista de Educação Física, 16(4), pp. 958-966 (2010)

4. Finberg, M., Braham, R., Goodman, C., Gregory, P., Peeling, P.: Effects of electrostimulation therapy on recovery from acute team-sport activity. Int J Sports Physiol Perform, 8(3), pp. 293-299 (2013)

5. Ciro, J. A. O., Rodríguez, M. P. C., E. A. V., Giraldo, S. P., Ching, I. C. G.: Lesiones deportivas. Iatreia Rev. médica Univ. Antioquia, no. 2, pp. 167-177 (2007) 
Miriam Reyes-Fernandez, Ruben Posada-Gomez, Albino Martinez-Sibaja, et al.

6. García-Soidán, J., Arufe-Giraldes, V.: Analysis of the most frequent injuries in tests of speed, half and long distances. Int. J. Med. Sci. Phys. Act. Sport, vol. 3, no. 12, pp. 260-270 (2003)

7. Arbizu, R. L. T., Raventós, K. E., \& Urrialde, J. M.: Actualización en el tratamiento fisioterápico de las lesiones ligamentosas del complejo articular del tobillo. Fisioterapia, 28(2), pp. 75-86 (2006)

8. CENETEC: Guía de Práctica Clínica del Diagnóstico y Manejo del Esguince de Tobillo en la Fase Aguda en el Primer Nivel de Atención. (2013)

9. Lisinski, P., Huber, J., Samborski, W., Witkowska, A.: Neurophysiological assessment of the electrostimulation procedures used in stroke patients during rehabilitation. The International journal of artificial organs, 31(1), pp. 76-86 (2008)

10. Amiridis, I. G., Arabatzi, F., Violaris, P., Stavropoulos, E., Hatzitaki, V.: Static balance improvement in elderly after dorsiflexors electrostimulation training. Eur. J. Appl. Physiol., vol. 94, no. 4, pp. 424-33 (2005)

11. Skahan, M. S., Lunau, K. R., Combs, D. K., Fischer, W. R.: Electrostimulation System. U.S. Patent Application 14/234,984 (2012)

12. Snyder-Mackler L., Delitto A. Bailey S.L. et al.: Strength of the quadriceps femoris muscle and functional recovery after reconstruction of the anterior cruciate ligament. J. Bone Joint Surg., 77- A(8), pp. 1166-1173 (1995)

13. Lieber R.L., Silva P.D., Daniel D.M.: Equal effectiveness of electrical and volitional strength training for quadriceps femoris muscles after anterior cruciate ligament surgery. J. Orthop. Res., 14(1), pp. 131-138 (1996)

14. Paternostro-Sluga T., Fialka C. Alacamliogliu Y, et al.: Neuromuscular electrical stimulation after anterior cruciate ligament surgery. Clin. Orthop. Rel. Res., Vol. 368, pp. 166-175 (1999) 